\title{
La ruse d'Ongmanpwa
}

Michel Cartry à l'écoute des documents ethnographiques des autres

\section{Odile Journet-Diallo}

\section{(2) OpenEdition}

\section{Journals}

Édition électronique

URL : http://journals.openedition.org/span/1710

DOI : $10.4000 /$ span. 1710

ISSN : 2268-1558

Éditeur

École pratique des hautes études. Sciences humaines

Édition imprimée

Date de publication : 1 janvier 2014

Pagination : $57-78$

ISBN : 9782909036434

ISSN : 0294-7080

\section{Référence électronique}

Odile Journet-Diallo, "La ruse d'Ongmanpwa », Systèmes de pensée en Afrique noire [En ligne], 19|

2014, mis en ligne le 05 février 2020, consulté le 10 février 2020. URL : http://

journals.openedition.org/span/1710 ; DOI : 10.4000/span.1710 


\section{La ruse d'Ongmanpwa}

\section{Michel Cartry à l'écoute des documents ethnographiques des autres}

\section{Odile Journet-Diallo}

Directrice d'Études EPHE Institut des mondes africains (IMAF)

Dans le récit gourmantché rapporté par Michel Cartry au début de sa contribution à l'ouvrage Tracés de fondation'

"Jaba, descendu du ciel sur une grande pierre, aperçut au loin, vers l'ouest, un homme également assis sur sa pierre, tout habillé de rouge. Assisté de son épouse, Ongmanpwa, Jaba procéda au rituel de libations de crème de mil dit yidi sala. Quand les puissances eurent reçu leur part, Jaba but une gorgée, Ongmanpwa également. L'homme habillé de rouge restait immobile. Jaba eut d'abord l'idée d'envoyer sa femme auprès de lui pour lui apporter la calebasse de sala, mais elle l'en dissuada et le convainquit d'appeler l'homme à venir partager la boisson sur le lieu du rite. Jaba ne se déplaça pas, l'homme rouge répondit à l'invitation, vint s'asseoir auprès de la calebasse et but une gorgée de liquide. Il fit ainsi acte de soumission.

Je ne reviendrai pas sur le fond de l'analyse que déployait Michel Cartry dans ce texte, écrit avec Danouta Liberski, consacré aux rites du bâtir dans leur rapport à la fondation et au statut rituel de personnages tels que le chef, le roi ou le maître de la terre, - analyse ailleurs commentée, notamment par Alfred Adler'2. Les premières pages de cette longue contribution - prélevées au titre qu'elles pouvaient se prêter à une évocation en temps limité -, je les prends simplement à la fois comme illustration de la manière qu'avait Michel Cartry d'organiser, de composer ses matériaux ethnographiques et comme métaphore du travail de déplacement auquel il conviait - et convie toujours - les ethnologues africanistes.

\author{
${ }^{1}$ Cartry \& Liberski, \\ 1990: 85.140. \\ ${ }^{2}$ Adler, 2010: 149.160.
}


Confrontant ce récit et ses différentes versions aux formes réelles de deux types de rituels : celui du yidi sala, effectué comme rituel d'hospitalité, de fondation d'une maison ou comme rituel public du chef, et celui de l'édification du vestibule du roi, avec ce qu'il suppose de précautions extrêmes à l'érection des poteaux des ouvertures de l'est et de l'ouest, Michel Cartry faisait apparaître la véritable nature de la "ruse " d'Ongmanpwa: son intervention permet non seulement à Jaba de prendre d'emblée l'ascendance sur un rival potentiel, mais aussi et surtout de conjurer la menace d'une confusion fatale entre lui et cet homme, dont Michel Cartry montrait qu'il est comme son double solaire, comme lui sorti, telle une pousse, de la terre, un " personnage en tous points semblable à soi-même mais qui, néanmoins, en différerait radicalement par un trait " (ici sa position cardinale : l'homme rouge assis à l'ouest figurant l'aspect à venir du soleil levant lorsqu'il sera sur son déclin).

Bien plus qu'une épouse avisée, Ongmanpwa est un "personnage qui, de façon prémonitoire, a vu le sort qui aurait échu à Jaba s'il avait rejoint son jumeau solaire ».

En explorant cette variation gourmantché sur le thème du double et des risques d'une confusion qui abolirait les distinctions, Michel Cartry dessinait un champ de questionnement immense qu'il aura développé ailleurs sous différents rapports: les montages de représentations et les rites liés au placenta, la figure du chef et de son double, la distinction des sexes dans ces moments rituels (funérailles, initiation) où elle semble abolie, suspendue ou subvertie.

Ce faisant, il indiquait là tout à la fois une manière d'articuler, dans un texte ethnographique, les faits de discours et la pratique rituelle, les gestes du " commencement " tels que les énoncent les récits mythiques et légendaires et les gestes rituels observés. Conduisant ses lecteurs et ses auditeurs dans les plis et les replis de ses enquêtes menées en terrain gourmantché, il avait l'art de leur faire partager le cheminement de ses réflexions et, corrélativement, une manière d'organiser des matériaux dont la composition, « à l'instar des casse-têtes chinois n'est possible que si chaque 
pièce, indispensable à l'ensemble, est située à une place différente de celle que sa forme initiale lui destinait " (ainsi qu'il l'écrivait à la fin de ce texte magnifique, "Les yeux captifs ${ }^{3}$ ", où les pièces à organiser étaient des morceaux du corps, l'œil, le sexe, le placenta).

Ce mode de composition supposait de savoir repérer les « lignes » qui vont et viennent d'un rite à l'autre à l'intérieur d'une même société et ce, en suivant le procédé même du rite, lorsque celui-ci induit chez l'observateur le retour d'une image qui en rappelle une autre et conduit cet observateur à changer de place ${ }^{4}$. Lui-même rompu à cet exercice, Michel Cartry savait en entrevoir les orientations possibles sur d'autres terrains.

Terrains proches, puisqu'il n'a cessé de pratiquer de manière intensive et systématique la comparaison avec les autres sociétés de l'aire mande-voltaïque; terrains africains plus éloignés à l'égard desquels il manifestait une curiosité sans cesse en éveil.

La force d'un questionnement ou d'hypothèses que les termes en lesquels ils étaient énoncés rendaient transposables sur ces autres terrains, l'invitation à " changer de place " est sans doute ce qui rendait si précieuse son écoute. Tous ceux qui ont eu la chance d'en bénéficier connaissent l'acuité toute particulière qui était sienne pour repérer dans un texte ou un exposé, les "zones d'ombre ", pour reprendre ses mots, d'une documentation, les failles d'un raisonnement, les sauts logiques, les associations et liaisons implicites avancées à l'insu de l'ethnographe. Documents déjà publiés, et là son érudition était sans faille, documents en cours de constitution que son questionnement invitait sans cesse à reprendre.

Cette attention aux matériaux des autres allait bien au-delà d'une seule lecture critique. À l'instar de l'intervention d'Ongmanpwa, il invitait chacun à bouger de son poste initial d'observation, à reprendre l'enquête, à recomposer ses matériaux, à quitter la pierre de ses premières interprétations. Michel Cartry, en quelque sorte, pensait les matériaux des autres.

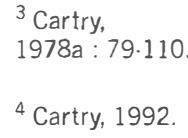


Travaillant dans une toute autre région d'Afrique de l'Ouest, en pays jóola, dans un groupe situé à la frontière du Sénégal et de la Guinée-Bissau, il n'est sans doute guère de pans de mes recherches qui n'aient, quelque part, été éclairés par cette écoute agissante. Je me permettrai d'en donner un exemple en évoquant, en filigrane, quelques-unes des pistes que ses relances (souvent initiées par cette formule qui était sienne: "ça m'a très fortement intrigué »...) m'ont permis d'esquisser. Du dossier par lui réuni sur le sacrifice, dossier imposant par sa force et sa densité, je n'évoquerai que quelques fils conducteurs, laissant ici dans l'ombre cet autre pan qui lui est intimement lié, celui de la divination.

Il serait facile de prendre la mesure des écarts qui peuvent séparer sociétés voltaïques et sociétés littorales des "Rivières du sud "(de la Gambie à la Sierra Leone)5 dont font partie les Jóola, à partir de quelques traits : l'inexistence, chez les Jóola, d'institutions de type maîtrise de la terre ou chefferie, les différences dans les modes de construction des liens au territoire, une partition générale de l'espace cultuel selon les sexes, une instance cosmique figurée non par le Soleil mais par le Ciel-Pluie, l'absence de notion aussi élaborée que celle de "parole " ou de "projet prénatal ", etc.

Pourtant, les questions posées par Michel Cartry et les relectures successives de ses textes n'ont cessé et ne cessent de m'ouvrir de nouvelles perspectives.

\section{Bref retour sur la question de la position des femmes sur la scène sacrificielle}

${ }^{5}$ Pour désigner cet ensemble de popula. tions de riziculteurs de mangrove d'implan. tation ancienne, les auteurs anglophones préfèrent le terme de Upper Guinea Coast, en opposition aux pays du golfe de Guinée, ou encore de Northern Rivers (vues de Freetown).
À l'époque où je rejoignais le laboratoire "Systèmes de pensée en Afrique Noire ", alors engagé, sous la houlette de Michel Cartry, dans un travail de longue haleine sur le sacrifice, les "zones d'ombre " de ma documentation en la matière étaient immenses. Outre des informations encore lacunaires sur la topographie des aires sacrificielles, la nature des biens sacrifiés et des puissances destinataires, le déroulement d'un sacrifice, je n'avais à apporter au débat collectif que ce fait, insolite du point de vue des sociétés voltaïques 
ou mandé, mais tout à fait ordinaire chez les Jóola, comme dans quelques sociétés voisines, de femmes maniant ellesmêmes le couteau sacrificiel et procédant à l'immolation de poulets, de porcs et parfois, en des circonstances particulières, de chiens. De manière générale, l'exclusion des femmes de la fonction de sacrificatrice dans de très nombreuses sociétés, en Afrique et ailleurs, est souvent rapportée à leur disqualification sociale dans le domaine public ou à l'incompatibilité, souvent énoncée, entre l'acte de mettre à mort et la capacité de mettre au monde. Que les femmes occupent la charge de sacrificatrice, comme officiantes dans les cultes à elles réservés, ou comme responsables de puissances auxquelles s'adressent les deux sexes, est cependant un fait banal pour les Jóola. Il semble pourtant paradoxal au regard des modes de la construction rituelle de la différence des sexes en cette société qui fait des formes d'écoulement sanglant un opérateur cardinal à l'établissement de toutes sortes de séparations et d'incompatibilités entre personnes, activités, espaces et temps.

Toute effusion de sang y est prise dans un faisceau d'interdits de lieu, de contact, de regard, qui sont autant d'interdits d'écoulement "sauvage " en des lieux ou des moments où il ne serait pas contenu dans l'espace et par le dispositif rituels adéquats. Ces interdits, qui, pour partie, ne sont pas sans rappeler les "tabous de la Terre " des sociétés voltaïques, relèvent entièrement de la juridiction de puissances de la terre appelées ukiin (sing. bákiin $\left.{ }^{6}\right)$, dont les autels quadrillent le territoire. Instances captées, plantées, génératrices d'interdits, de trajectoires et d'attaches spécifiques, elles sont souvent subsumées, par synecdoque, et pour éviter de les nommer, sous le terme de etaamay, "la terre".

La première ligne de séparation instituée par ces interdits est celle des sexes telle qu'elle est façonnée, en plusieurs étapes, dans l'espace rituel et symbolique. Les deux temps forts de ce façonnement en sont l'initiation masculine ${ }^{i}$ et, pour les femmes, le premier accouchement. De la transformation rituelle d'une promotion de garçons en hommes, le principal effet est de doter les initiés de la capacité à faire

\author{
${ }^{6}$ Le même terme \\ désigne à la fois \\ l'instance, le sanctuaire \\ et l'autel. \\ 7 «Tournant» de village \\ en village, l'initiation \\ la plus répandue, le \\ bukut, a lieu tous les \\ trente ans. Dans les \\ villages non islamisés, \\ la réclusion des initiants \\ débute par l'opération \\ de circoncision. \\ Celle-ci n'était pas \\ pratiquée autrefois, \\ mais on faisait couler \\ le sang des initiants par \\ une incision à l'aine.
}


8 Dès leurs premières règles, jeunes filles et femmes mariées vont en ces périodes passer la nuit dans la hutte collective édifiée dans chaque sous-quartier. Après un accouche. ment, une femme ne peut rejoindre la maison maritale que quelques semaines plus tard.

${ }^{9}$ A. Zempleni, $1991: 3$. couler le sang, notamment hors contexte sacrificiel, à se marier et à manipuler des cadavres. Du côté des femmes, c'est le premier accouchement d'un enfant vivant dans la case d'accouchement traditionnelle - case implantée dans chaque quartier à l'écart des habitations et dont à l'époque, faute d'avoir été invitée à y pénétrer, je ne savais pas encore qu'elle était construite comme un véritable sanctuaire - qui les fait accéder au statut d'adulte et à la position d'actantes sur la scène sacrificielle. Ces deux moments rituels, par le traitement des substances ou des éléments chus du corps en des lieux directement placés sous la tutelle d'ukín villageois, ont en commun d'attacher, par des liens indéfectibles, chaque natif du village à sa " terre".

Sur la scène sacrificielle, ce qui est discriminant pour les femmes, quelle que soit leur position, n'est pas, comme en bien d'autres sociétés, leur capacité à procréer (il faut, au contraire, qu'elles en aient fait la preuve et elles peuvent officier bien avant d'être ménopausées), mais les périodes où elles saignent (menstrues, suites de couches): elles doivent se tenir à l'écart de leurs propres ukíin, mais aussi de leur maison $^{8}$ et des rizières. Le sang féminin est réputé mettre en péril la puissance sexuelle des hommes, mais aussi la fertilité des autres femmes comme des terres cultivées.

En un premier temps, suivant en quelque sorte la pente de la littérature ethnographique et des spéculations jóola relatives aux épanchements sanglants, ce sont ces deux moments - initiation masculine et premier accouchement - dont j'avais privilégié le rapport, dans la foulée de cette observation récurrente, rapportée par maint ethnologue, que les initiations masculines " tribales " réalisent la séparation sociale des sexes par «l'appropriation mimétique des propriétés ou des capacités naturelles de l'autre sexe ${ }^{9}$ ". Les faits jóola, et notamment la manière dont est traité le premier accouchement, empruntant à l'initiation toutes ses caractéristiques séquentielles et sa violence rituelle, ne pouvaient qu'apporter de l'eau au moulin. Il ne s'agissait que d'analyser les singularités de cette forme d'appropriation qui ici, semblait réciproque: circulation de signifiants, rapprochements sémantiques, homologie des interdits alimen- 
taires et posturaux, mise à mal des corps, barrières du secret, etc. Mais Michel Cartry me l'avait vite fait remarquer :

"C'est plus qu'un rapprochement, c'est quelque chose de l'initiation et de l'accouchement ${ }^{10}$."

Qu'en était-il de ce "quelque chose " de l'initiation et de l'accouchement ou, en d'autres termes, quel même questionnement traversait ces deux rituels? Qu'est-ce que cela avait à voir avec le schéma sacrificiel ?

La participation aux ateliers du laboratoire et la lecture des premiers numéros des Cahiers Systèmes de Pensée en Afrique Noire consacrés au sacrifice, m'avaient très vite obligée à reprendre de nouvelles enquêtes sur ce rite principiel qu'est le sacrifice chez les Jóola ${ }^{11}$ et à étudier de plus près la complexité des découpages d'une géographie religieuse - tout entière articulée autour de ces sanctuaires et de ces autels dûment "plantés " que sont les ukiin -, à affiner les distinctions entre différents types de sacrifice et réexaminer les variations observées dans leur déroulement ainsi que les singularités des sacrifices exécutés par les femmes. Outre la mise à l'écart périodique, ces singularités s'observent dans les postures (les femmes sont toujours assises à l'équerre à même la terre alors que les hommes utilisent des troncs équarris ou des tabourets), les modes d'adresse à l'instance (simplement saluée dans les ukiin de femmes alors que du côté des hommes, il faut la convoquer, s'assurer de sa présence à plusieurs reprises), les instruments cultuels (pour les libations, une louche-calebasse pour les femmes, un grand coquillage régulièrement frappé sur le bord de l'autel pour les hommes), l'exécution du rite de sortie de l'aire sacrificielle: les officiantes sont beaucoup plus minutieuses dans ce travail qui comporte des gestes d'onction, avec le vin de palme consacré mêlé, le cas échéant, au sang de l'animal sacrifié, de différents segments du corps du sacrifiant (tête, visage, paumes, ventre, dos, mollets). Dans tout bákiin, ce geste obligatoirement effectué lorsque l'on quitte l'aire sacrificielle semble avoir pour fonction de transformer une emprise diffuse, aux effets imprévisibles, en une protection individualisée et dirigée. Dans l'espace des ukíin féminins, tout se passe comme si cette alchimie sacrificielle nécessitait

\author{
10 Dans l'une de \\ ces conférences, il \\ rappellera, à propos \\ de cette autre forme \\ de rapprochement que \\ I'on peut opérer entre \\ funérailles et initia. \\ tion: «Comparer les \\ funérailles et l'initia. \\ tion ne consiste pas \\ à se demander si les \\ éléments d'un rituel se \\ retrouve dans l'autre, il \\ s'agit plutôt de recher. \\ cher si ce n'est pas un \\ même questionnement \\ qui traverse ces deux \\ rituels, questionnement \\ auquel il ne sera jamais \\ donné réponse mais \\ dont les termes, d'un \\ rite à l'autre, entrent \\ dans des combinaisons \\ différentes. ॥ Cartry, \\ $1989: 91$ \\ 11 C'est par le terme \\ " gens du sacrifice " que \\ les Jóola attachés aux \\ cultes des ukiin \\ se distinguent \\ des convertis à I'islam \\ ou au christianisme.
}


un surcroît d'attention. Mais ce qui, à l'époque, allait véritablement forcer ma réflexion, c'est cette hypothèse lumineuse qu'avait formulée Michel Cartry à propos des faits gourmantché, d'une affinité entre le schéma sacrificiel et le modèle de la naissance.

Au-delà de l'apparence (" une femme si elle est à genoux sur la terre, elle ressemble à une morte »), Michel Cartry invitait à considérer que s'il y a bien dans tout accouchement une victime, c'est le placenta, cette part de lui-même que l'enfant perd à sa naissance, ce compagnon "plus luimême que lui-même " auquel, pour vivre, il doit renoncer: "L'immolation, écrivait-il, loin d'être un meurtre, est comme la reproduction du geste de la sage-femme, lorsque, coupant le cordon, elle sépare l'enfant d'avec son placenta ${ }^{12}$ ".

À la différence de nombreuses sociétés d'Afrique de l'Ouest, les Jóola ne considèrent pas le placenta comme double ou comme jumeau de l'enfant. La gémellité y est d'ailleurs considérée de manière générale comme une affliction. Le placenta est désigné comme une part de la mère - " la mère de lui (l'enfant)» -, ou encore comme son assise - "le tabouret " - : "être assis ", cette formule ne réfère pas tant à une posture, qu'à une forme de présence totalement intégrée à l'espace-temps environnant. Il est précieusement recueilli par l'une des officiantes - désignées comme "celles qui vont enterrer la chose la nuit " - du plus important bákiin détenu par les femmes, le karaay, et emporté dans un grand canari pour y être enfoui secrètement avec tous ceux des enfants nés dans un même quartier. Afin de ne pas "fermer " le pouvoir de procréation de la mère, on le dépose en terre la face maternelle tournée vers le bas et la face foetale vers le haut. Support de l'assise initiale du nouveau-né dans ce monde, ce " tabouret " chu du corps de la mère, sorte de mère du dedans, renforce la puissance de cette instance - qui préside aux naissances et de manière générale à la fertilité des terres et des femmes -, en même temps qu'il attache définitivement l'enfant à la terre qui l'a vu naître. C'est dans ce même bákiin que les femmes se rassemblent pour les sacrifices qui « ouvrent » et "ferment " chacune des opérations agraires dont elles sont 
exclusivement chargées, l'une comportant un délicat transfert des plants (repiquage), et l'autre, une brutale séparation d'avec la terre (récolte).

Mais si les termes et les montages de représentations permettant de penser l'affinité sacrifice/naissance étaient différents, il m'apparut que le modèle de l'accouchement et de la naissance tels qu'ils sont rituellement construits par les Jóola, condense, en un raccourci saisissant, les processus qui s'enchaînent dans tout sacrifice : permutation des rôles (la parturiente étant tour à tour placée en position de victime et de sacrifiante), état de vacillement (on dit d'une femme qui accouche que "Dieu l'a appelée " et qu'elle a un pied dans la tombe), silence requis lors de moments critiques (dans le temps qui suit l'expulsion du bébé comme dans celui qui suit l'immolation de l'animal), perte, et inéluctabilité du mouvement enclenché par les premières libations de vin de palme enchâssées dans les formules d'appel adressées à la puissance.

Dès lors que l'officiant(e) a commencé à verser avec son coquillage ou sa louche calebasse les premières gouttes de vin de palme sur l'autel, tout ce qui a été apporté dans le sanctuaire est désormais consacré au bákíin et n'en pourra ressortir : il en est de même de l'écoulement du liquide amniotique qui, s'il se répandait dans la maison, mettrait celle-ci tout entière sous l'emprise du bákíin karaay. Les responsables emportent alors le riz et les pagnes qu'elle contenait dans l'enceinte du sanctuaire. Si la parturiente perdait ne serait-ce qu'une goutte de sang dans la maison, celle-ci serait incendiée. Les faits jóola invitaient à considérer l'accouchement lui-même comme le référent implicite du rite sacrificiel.

La logique du sacrifice sanglant pratiqué par les femmes n'en restait pas moins problématique. Si le sacrifice masculin prend place parmi une série d'actes pensés comme homologues (chasse, guerre, labour des rizières, récolte du vin de palme ${ }^{13}$ ) mais dissociés les uns des autres, pratiqué par les femmes, il s'inscrit dans le prolongement d'événements vécus individuellement et dont le déclenchement échappe à tout contrôle ${ }^{14}$.

\author{
13 Lors des labours, \\ la terre est réputée \\ "saigner "; le vin de \\ palme recueilli par \\ incision des nervures \\ en haut de l'arbre est \\ considéré comme le \\ sang du paimier.
${ }^{14}$ À différentes reprises, Françoise Héritier avait formulé l'hypothèse que le ressort fondamental de tout le travail symbolique greffé sur le rapport des sexes serait cette opposition maîtrisable/non maîtris able, voulu/subi, relative aux écoulements de substances corporelles (sang, sperme, lait notamment). Dans l'ouvrage qu'elle publiait en 2002, elle revenait sur cette hypothèse: faire de l'opposition maitri. sable/non maîtrisable le ressort fondamental de la hiérarchisation entre les sexes serait, disait.elle, supposer comme existant au préalable une valorisa. tion du vouloir, de l'actif sur le passif. Cette remise en question de la valorisation de l'un sur l'autre laisse cependant posé comme évident le fait que tout écoulement de sang menstruel soit pensé comme "passif ».


Là encore, les remarques de Michel Cartry m'ont vite permis de reconsidérer cette opposition première. Il m'avait rappelé à cette occasion les passages où Victor W. Turner se demandait pourquoi les femmes traitées pour des dysménorrhées étaient identifiées à des hommes qui répandent le sang. Les détails du rite laissaient supposer à Turner que pour les Ndembu, une femme, en perdant son sang menstruel, renonce de façon active au rôle maternel et nourricier que l'on attend d'elle. Elle se comporterait alors comme un homme (chasseur ou homicide) qui tue ${ }^{15}$. Si, à ma connaissance, les faits jóola ne permettent guère de formuler une telle interprétation, on peut par contre remarquer que dans le temps même où les écoulements de sang surviennent en elles, les femmes deviennent les actantes de leur transformation rituelle. Immédiatement réinscrits dans le temps et l'espace codés d'une relation aux puissances de la terre, elles en dirigent les effets par l'observation des interdits et des rituels appropriés. Par ailleurs, en déplaçant l'attention vers d'autres rites, tels par exemple ceux qui traitent des meurtriers ou des parents de jumeaux, se dessine une tout autre manière de penser le rapport entre état et action, être et faire. Ici, le traitement rituel des différents écoulements sanglants comme de ceux qui les provoquent, les subissent ou encore entrent à leur contact par un simple regard, ne tientguère compte des oppositions volontaire/involontaire, actif/passif : ce que révèlent d'ailleurs les faits de langue lorsqu'ils nomment par un même terme un rite et l'état ou le statut de celui qui l'effectue (ainsi des séquences funéraires désignées par le même mot, arimenew, que les femmes natives du lignage qui en sont chargées, ou encore l'état de parent de jumeau, les afflictions et le bákíin qui en traite, appelés du même terme, bulunt). Le fait que les femmes voient couler leur sang alors que les hommes le font couler à la guerre ou à la chasse, n'explique rien de la position des unes et des autres sur la scène rituelle (et plus généralement d'ailleurs, sur la scène sociale).

Une nouvelle piste de réflexion, encore loin d'être aboutie et que je n'indiquerai qu'en filigrane, allait s'ouvrir lorsque plus tard Michel Cartry, à de nombreuses 
reprises, me demandait de revenir sur les particularités de la destruction sacrificielle (" ce n'est pas un détruire comme un autre ", insistait-il). Au fil de ses articles sur le sacrifice, lui-même avait consacré de longues pages aux modalités de cette destruction, en analysant les différentes séquences de cette catégorie de sacrifice qu'est le "sacrifice d'attache " chez les Gourmantché. Transformation complète du corps de l'animal sacrifié en " animal-signe ", jusqu'à l'arrachement systématique à sa dépouille de tout ce qui "gardait les attributs de la vie ", sacrifice de l'œuf dont le sacrificateur prélève en plusieurs fois la substance qu'il répand sur le bulo, bris du fragment de calebasse sur lequel étaient gravés les signes tracés par le devin, autant de moments dont il a cherché jusqu'au bout à dégager toutes les implications. De toutes ses minutieuses analyses, je ne prélèverai ici que l'un des fils en m'appuyant sur ce moment très particulier lors duquel l'officiant, dans les derniers moments du rituel, brise et rebrise, - " comme s'il était animé d'une sorte d'acharnement à détruire " ${ }^{16}$, le morceau de calebasse couvert de signes. Cet étrange traitement, il le qualifiait d'opération de "réduction " (au sens " de remise en place ", précisait-i ${ }^{17}$ ). "Mais l'objet une fois brisé n'est pas mis au rebut. Pièce par pièce, la chose brisée est collée sur l'objet matérialisant le bulo, comme si l'on y déposait une nouvelle offrande ". Au terme du sacrifice, les signes doivent être renvoyés au tout dont le fragment était issu et cela par le biais d'une réduction. "Le bulo ira porter les signes à Dieu, il lui présentera ce qui a été sacrifié, mais le sacrifice ne sera accepté que si Dieu reconnaît dans ce qui lui est présenté son inscription originelle "(idem.). En effectuant ces gestes, l'officiant prononce des formules exprimant des vœux de mort contre l'ennemi. Pourtant, poursuivait Michel Cartry, ce n'est pas le thème de la destruction qui domine dans le type de discours énoncé par ses informateurs à propos de cette séquence. Ces derniers "invitent à prêter attention au contraste suivant. En "attachant" le morceau de calebasse, il [le devin] a œuvré pour lier ensemble les signes premiers du monde et les signes du destin individuel de quelqu'un, le consultant $[. .$.$] . En mettant en pièces la tablette, l'offi-$

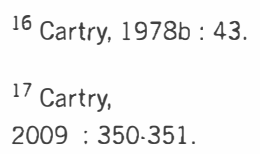


ciant accomplirait un acte dont la finalité serait de dissocier et de libérer les signes attachés ». Dans son article de $1981^{18}$, il avait déjà développé les implications du choix " fait par les Gourmantché d'inscrire la formule singulière d'un rite sacrificiel " sur cet éclat détaché de la coque du fruit du calebassier, lequel, en bien des rites, est assimilé au "ventre du monde ", à la matrice cosmique originelle, où est "gesté " tout ce qui naît ici-bas. Les sillons de la surface interne de cette coque - que le devin va gratter et poncer pour les faire apparaitre-, sont comme les signes premiers du monde, les signes du commencement, des signes toujours en mouvement.

"Ce qui ly] est écrit, ce n'est pas seulement l'évolution du fruit du calebassier, mais le devenir même des signes du monde ${ }^{19}$. "

Ils commandent la "descente " du tagama, ce " pouvoir de créer ou de procréer " qui siège dans le ventre du monde.

"Il faut donc que, pour chaque rituel, les acteurs se connectent à cet univers des signes premiers et y ajustent leurs gestes, leurs paroles et leurs actes ${ }^{20}$."

Michel Cartry précisait alors la différence d'enjeu entre les deux types de sacrifice dûment distingués par les Gourmantché, le "sacrifice cérémoniel " et le "sacrifice d'attache ". Le premier est intégré à des cérémonies collectives qui se déroulent pendant ces jours particuliers du calendrier lunaire "que Dieu a séparés pour lui seul ", lesquels correspondent à un moment du temps cosmique, celui de la naissance, " un temps où la chose réalisée s'achemine déjà vers sa destruction ". Dans ce type de sacrifice, il n'y a ni consultation divinatoire préalable, ni extraction, du corps d'un animal-signe, de "mots de passe " avec lesquels on construit une demande faite au bulo. Le déroulement de tous les actes rituels est en quelque sorte dicté par un schéma d'action préétablie, fixée, dans une sorte de " code liturgique " qui s'accorde au rythme de travail de la matrice

${ }^{18}$ Cartry, 1981

$19 / \mathrm{dem}: 210$.

$20 /$ dem : 211 cosmique. Le "sacrifice d'attache "implique au contraire que l'on interroge la terre sans relâche pour faire apparaître les instances et les ancêtres qui "sont en rapport avec le consultant ". Il faut, pour celui-ci, retrouver l'attache, « réali- 
ser une jonction, une rencontre avec lui-même comme futur sacrificateur et le bulo auquel il destine ses offrandes ${ }^{21}$ ". $\grave{A}$ propos du commentaire de ses informateurs : "Si tu ne brises pas le morceau de calebasse, le bulo refuse tout ce que tu lui as transmis ", Michel Cartry terminait son dernier article par cette question :

"Faut-il interpréter une telle déclaration comme si, par ce bris, l'officiant renforçait le lien de proximité avec la puissance " destinataire ? ${ }^{22} "$

C'est cette question qui m'invite aujourd'hui à reprendre le fil de réflexions jusqu'alors laissées en suspens. Les modalités du sacrifice jóola, éminemment diverses selon la nature de l'objet traité, les circonstances, la catégorie de puissance sollicitée sont difficilement comparables aux faits gourmantché, et il ne peut ici être question d'ouvrir un tel dossier. Rappelons simplement que, bien que les Jóola ne les distinguent pas dans la langue, la différence entre " sacrifices cérémoniels " et " sacrifices d'attache " pourrait avoir son équivalent dans celle qu'ils établissent entre sacrifices calendaires ou encore liés au cycle de vie, d'une part, et sacrifices individuels effectués après consultation divinatoire, de l'autre. En fait, une telle distinction est déjà dûment marquée, en amont du sacrifice, par la séparation entre deux catégories d'ukiin : les uns traitant de la procréation, de la fertilité des terres et des humains, de la séparation des sexes et de son analogon cosmique, la séparation et l'alternance des saisons sèche et pluvieuse ; les autres, de ce que l'on pourrait dire, pour faire vite, des " affaires " de la vie en société. Les sacrifices dus aux premiers - au premier chef, les sacrifices liés au cycle agraire - obéissent pour l'essentiel à un " code liturgique " fixé d'avance. Leurs desservants sont capturés contre leur gré, lors de cérémonies calendaires, tandis que les autres accèdent à leur charge par de lourdes tournées sacrificielles; le lien qui les unit à leur puissance n'est pas de même nature ${ }^{23}$. Pour être initié aux fonctions de devin, il faut préalablement avoir acquis l'un des ukiin spécialisés dans la divination.

Était-il possible de repérer un tel processus de réduc-

${ }^{21}$ Cartry, $2009: 341$.

22 Idem : 351.

${ }^{23}$ Voir Journet, 1993 
24 Je me permets de renvoyer à un article antérieur: "“Prendre un bákiin sur le dos" ...] : Journet.Diallo, 2009 : 111.136 . fice jóola et plus particulièrement dans celui exécuté par les femmes? Il serait beaucoup trop long ici de reprendre les différents moments au terme desquels, lors de tout sacrifice, la substance vitale des biens sacrifiés mais aussi certains des pans de la vie du sacrifiant, enchâssés dans le flux de paroles adressées à l'instance, sont renvoyés, non à Dieu, mais à la "terre ", une terre qui imprime à l'espace sa géographie première et n'est morcelée ni par les différenciations généalogiques ni par les avatars de l'histoire locale, une terre en laquelle transitent les ukiin et les " âmes " du riz et des défunts à renaître.

Dans la description des opérations de séparation, d'arrachement, de morcellement, de bris puis de " collage ", qui s'enchaînent en divers moments du sacrifice gourmantché, une image revient souvent sous la plume de Michel Cartry, celle d'un " acharnement à détruire ». Cette image, on pourrait la retrouver dans différents actes rituels jóola, comme celui, par exemple, qui se déroule après la mort d'un devin-guérisseur lorsque son sanctuaire personnel et tous les objets par lui installés sont fracassés au sol et proprement cassés en petits morceaux. Cet acte parachève la déliaison du détenteur défunt d'avec son bákiin ${ }^{24}$. Cependant, laissant ici de côté le champ des rituels divinatoires et sacrificiels relevant de cette catégorie d'ukiin, c'est vers d'autres moments rituels que je tournerai le regard, des moments singuliers oủ ce qui est visé par cet acharnement, ce ne sont ni des objets, ni des supports de signes, mais des corps féminins.

Le cas le plus extrême, que je ne ferai qu'évoquer, est celui du traitement d'une femme morte enceinte ou en couches. Dans l'espace protégé d'une épaisse palissade où est construite la case d'accouchement - de forme ronde comme seules le sont les cases vouées à un bákiin - sont plantés les autels de deux ukíin : celui qui préside à l'accouchement et à la naissance, erumun, à l'intérieur de la case et un deuxième, à l'extérieur, appelé erípay (substantif tiré de rip ou rúp, "arracher ", "tirer "). Ce qui est mis sous la tutelle de ce dernier, c'est ce qui sera dévoilé à une jeune mère, dans les semaines qui suivent son premier accouchement lors- 
qu'elle ira offrir un important sacrifice au karaay, ce bákiin, rappelons-le, qui intervient dans toutes les opérations mettant en jeu un processus de procréation. Ce sacrifice effectué avec du vin de palme, de la farine de riz et un porc, signe son intégration aux associations cultuelles liées aux ukín féminins. Le dévoilement du secret de eripay, achève le processus qui aura fait d'elle une mère socialement accomplie. Or ce secret renvoie précisément à ce qui représente l'échec le plus flagrant, le "ratage "le plus complet du processus de procréation: le décès d'une femme enceinte avant l'expulsion de l'enfant. Chez les Jóola, comme en bien d'autres sociétés africaines, une telle mort est considérée comme une calamité collective, propre à signer la fin de toute procréation. On ne peut ici s'empêcher de penser à cette observation formulée par Henri Hubert et Marcel Mauss, à propos du sacrifice :

"Á partir du moment oủ il est commencé, il doit se poursuivre jusqu'au bout sans interruption et dans l'ordre rituel. [...] Les forces qui sont en action, si elles ne se dirigent pas exactement dans le sens prescrit, échappent au sacrifiant et au prêtre et se retournent contre eux, terribles ${ }^{25}$."

Pour contrecarrer la lourde menace qui pèse sur le village, les officiantes vont d'abord œuvrer à reprendre, pour le mener à son achèvement, le processus brutalement interrompu par la mort de la parturiente, un processus qui, ordinairement, ne relève ni d'elles, ni de la mère, mais de l'instance karaay. Le corps de la mère, directement amené dans l'enceinte de la maternité traditionnelle au cas où elle serait décédée ailleurs ${ }^{26}$, va être l'objet d'opérations complexes. La responsable de eripay, assistée des officiantes de erüun et du karaay, devra se livrer à un travail, décrit par les opératrices elles-mêmes comme terrible, pour aboutir à l'extraction du fœetus - par basiotripsie ou par césarienne post-mortem, à l'aide d'une hachette, d'une flèche et d'un poignard - et, dans la foulée, du placenta. Le petit cadavre sera enterré dans l'enceinte du bákíin, avec tous les enfants morts pendant la réclusion de la mère dans la maternité, le placenta dans le karaay du quartier, et le corps de la mère, dont le ventre aura été bourré de bandes de pagnes funéraires, sera interrogé sur place avec virulence : qu'a-t-

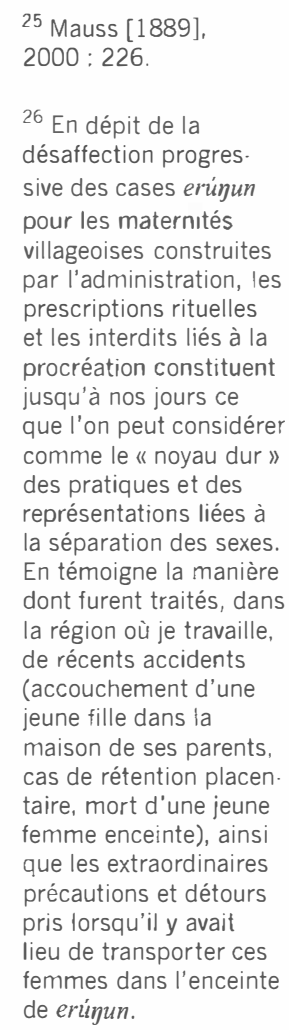


27 Dans d'autres groupes jóola, ce sont les femmes qui se chargent d'aller secrètement l'inhumer en forèt. elle fait pour que karaay la tue? Au crépuscule ou à l'aube, sa dépouille sera rendue à ses parents pour être très rapidement enterrée, avant même que n'éclatent les lamentations funéraires, à un emplacement réservé du cimetière du sous-quartier ${ }^{27}$. Aucun des rites funéraires ordinaires ne sera exécuté. Le placenta, les dépouilles de la mère et de l'enfant sont mis en terre, en trois lieux séparés, chacun voué à des destins différents. Le corps de la mère a été définitivement réduit à son état de tombe initiale de l'enfant et du placenta : les pagnes funéraires qui emplissent la place béante laissée par l'extraction du foetus et l'arrachement de l'amnios emportent la marque de la fin prématurée d'un procès de procréation. Mais cette autre part de la mère qu'est le placenta, doit retourner au karaay lequel réclame, pour être apaisé, un très lourd sacrifice (aux frais du père de l'enfant, tenu de fournir aux officiantes tous les biens nécessaires à sa réalisation, vin de palme, poulets, gerbes de riz en quantité et un gros porc).

Autre cas d'échec du processus de procréation, la stérilité d'un couple ou la mort répétée des enfants qu'une femme met au monde, donne également à voir, cette fois de manière publique, un acharnement sinon à détruire, mais à briser, à dépouiller le corps d'une femme de tous les attributs de son identité sociale, voire humaine. Lors du traitement rituel auquel est soumise une mère en mal d'enfants, elle subit une série d'épreuves infligées par ses aînées dans le rite. Contrainte, de gré ou de force, de quitter la maison et le quartier marital, on lui attribue un nouveau nom, un nom de dérision, on la traite comme un animal, on l'attife de loques, on l'insulte, on l'humilie, on la frappe, on se rit de ses souffrances... Les différentes lectures que j'ai pu en développer ailleurs laissaient toujours une part d'énigme: en quoi cette mise à mal, cette obstination à la réduire à l'état de " chose vivante ", dénuée de toute subjectivité, de toute dignité, était-elle censée agir sur le processus de procréation?

D'autres moments rituels, aux marges de la scène sacrificielle, ont en commun de mobiliser les corps féminins comme instruments d'une mise en rapport avec l'espace des puissances invisibles: plongées collectives, nues, dans 
l'eau d'un bolon en cas d'épidémies, nuits passées dans les sanctuaires lorsque la mort menace, danses interminables et obligées pendant des jours à l'occasion de certaines funérailles, cette dépense d'énergie étant posée comme équivalente à des sacrifices auxquels le village n'aurait pu faire face. Lorsque la pluie se fait rare ou tarde à venir, lorsque les chenilles dévorent le riz des pépinières, compromettant toutes les récoltes à venir, c'est encore à elles de s'exposer pour interpeller Emitey, le Dieu du ciel et de la pluie lors de longues processions ou de voyages de type chamanique où elles montent le visiter. Ces voyages, décrits comme de périlleuses ascensions, sont préparés lors d'un rituel, organisé sous l'égide d'un bákiin villageois, qui met en scène un dispositif sacrificiel singulier. Une fosse profonde, appelée " tombe ", est creusée dans le sable de la place de chaque sous-quartier: pendant six jours, on y versera des centaines de litres d'eau puisée à proximité des rizières, les parts sacrificielles réservées au bákíin (sang, vin de palme, morceaux de viande), ainsi que tous les restes (os, écailles, eau de vaisselle, etc.) de la cuisine collective qui se déroule sur la place. Lorsque la fosse commence à déborder, certaines femmes, "tombées" en transe, vont plonger la tête et les mains, se laver et s'abreuver longuement dans ce qui, au fil des jours, est devenu un véritable cloaque. Imprégnées du produit déjà consacré de tous les sacrifices préalables, tout se passe comme si, en fermant la boucle d'une mécanique sacrificielle poussée à son point ultime, elles se trouvaient ellesmêmes projetées, comme ces restes, aux confins du monde des vivants et de celui de l'invisible. C'est cette immersion initiale qui les rendra aptes à partir un peu plus tard visiter Emitey.

Quelles qualités particulières seraient donc attribuées aux femmes dans l'établissement d'une relation avec les puissances de la terre ou du ciel, pour que leurs corps puissent être manipulés comme instruments rituels, voire comme matière oblatoire, et qu'elles soient considérées comme ultime recours lorsque la vie vacille ${ }^{28}$ ? Dans la pensée jóola, les passages entre le monde des morts et des entités invisibles d'une part, et celui des vivants, de l'autre, se réalisent dans

\footnotetext{
28 Commentant le

dicton jóola: "C'est la femme qui éteint le feu », Nazaire Diatta écrivait: " II arrive que des situations échappent complète. ment au contrôle des hommes surtout quand Il s'y mêle du métaphy. sique. Alors

on se tourne vers les femmes $»$ 1998 : 174.
} 
${ }^{29}$ Ce récit que j'ai recueilli sur mon terrain en Guinée-Bissau, est très largement répandu dans tous les groupes jóola. Il a également été rapporté par deux autres auteurs: N. Diatta, $1998: 18$ et M. R. Schloss, $1988: 31 \cdot 32$. Je reprends ici la version de ce dernier qui est la plus développée. des espaces de transition: le monde aquatique et le monde souterrain sont les lieux où transitent les âmes des morts à renaître, les doubles animaux, les génies et où résident les ukiin. D'accueillir en leur sein les défunts en voie de réincarnation et d'avoir à frôler la mort en laissant à la terre une part d'elles-mêmes chaque fois qu'elles accouchent, les femmes seraient créditées d'une aptitude particulière à affronter ces espaces indistincts où s'ouvrent les brèches entre le monde des vivants et celui des instances invisibles. Mais si cette aptitude qui leur est conférée explique qu'elles soient mobilisées lorsque la survie du groupe est en jeu, elle ne suffit pas pour autant à comprendre certaines singularités des rites qu'elles accomplissent, sur la scène sacrificielle ou ailleurs.

Un récit jóola, relatif à l'origine de la maison et du mariage ${ }^{29}$, permet sans doute de poser autrement la question, en laissant entrevoir la façon dont les Jóola se représentent le rapport originel des femmes à la terre.

"Quand Dieu envoya l'homme et la femme sur la terre, la femme creusa un trou dans lequel elle s'installa. L'homme lui dit qu'il allait la rejoindre là, mais elle refusa. Alors l'homme lui dit : "Ah, ne nous a-t-il pas dit de nous installer là-dedans?". Mais la femme dit : "Non, je ne veux pas ", et elle frappa l'homme. En ce temps, l'homme n'avait pas de forces dans ses mains, rien. Oui, il n'avait pas de mains. Après un moment, Dieu descendit leur rendre visite. Il trouva l'homme complètement assommé. L'homme lui dit : "Elle m'a tué, l'autre personne, tu as dit qu'elle était bonne, mais quand je suis allé pour rester avec elle, elle a refusé et m'a frappé. Oủ vais-je demeurer ? ». Dieu appela la femme et lui dit de s'asseoir avec l'homme. La femme dit : "Comment? Il n'est pas bon, il veut voir ce que je fais ". Dieu lui dit d'approcher. Alors, il lui prit les mains et tira, tira. Elle n'avait plus aucune force. Dieu appela l'homme : "Je vais te montrer la maison. Tu construiras une maison ". Il dit à l'homme de puiser de l'eau et celui-ci se mit à construire. Il bâtit et bâtit. Il alla chercher du bois et construisit le plafond. Dieu lui dit : "Dépêche-toi, la pluie va bientôt tomber. Quand elle tombera, la femme viendra et restera avec toi ici.- Elle qui a essayé de me tuer ? Jamais ! - Non, non, attends! ". L'homme alla aux champs et rapporta de la paille. Il coupa de la paille et la lia, puis Dieu lui dit : "Couvre vite la maison, la pluie arrive ". Il plut durant trois jours. Il plut, il plut, il plut. L'eau entrait dans le trou de la femme. La femme sortit en courant du trou en disant : "Ouah ! où vais-je demeurer maintenant? ". Elle alla trouver l'homme : "Je veux rester ici ". Mais 
l'homme refusa : "Non, tu m'as chassé de ton trou ; maintenant notre père m'a montré cette maison et m'a dit de rester ici ». La femme rétorqua : " $\mathrm{Ha}$ !, comme je suis plus forte que toi, nous allons nous battre aujourd'hui ". Ils se battirent, l'homme la frappa et la chassa.

La femme partit se plaindre à Dieu : " La pluie est venue et a ruiné ma maison, ce n'est pas bon ". Dieu lui dit : "Mais n'y a-t-il pas ici l'autre personne? " Elle répondit : "Oui, elle est ici, mais quand j'ai dit que nous allions rester ensemble, elle a refusé. Nous nous sommes battus et elle m'a frappée jusqu'à ce que mon corps devienne brûlant ! " Dieu lui dit : "Retourne ; dis-lui que j'ai dit qu'elle devait vivre avec toi, que je vous ai envoyés du ciel sur la terre pour que vous ayez une personne comme moi, je vous ai eus. "Elle partit, répéta cela à l'homme, mais il refusa. Elle retourna vers Dieu qui lui dit d'essayer à nouveau, mais l'homme refusa encore. Quand elle revint, Dieu lui dit de s'asseoir. Elle s'assit. Il lui dit : "Prends ce chapelet de grains et attache-le autour de tes hanches. ". Elle attacha le chapelet. "Regarde là encore, quels grains sont les plus beaux? ". Elle choisit d'autres grains encore et les attacha aussi. Dieu lui dit : "Retourne à nouveau vers lui, s'il refuse, va vers lui et fais résonner les grains avec tes mains. Ils résonneront, yiiss. Alors, tu resteras. ". Elle partit et quand l'homme la chassa, elle fit résonner les grains... yiiss. L'homme l'appela : "Reviens, reviens ! - Mais tu m'as dit de partir ! - Non, non, reviens! ». Elle revint, ils s'étendirent ensemble. Le mariage vient de là.

Il y aurait beaucoup à dire de ce récit ; je me contenterai ici d'en tirer brièvement deux remarques. Ce qui distingue les " deux personnes " jetées par Dieu sur la terre, est entièrement contenu dans leur mode d'habiter: une femme forte, se suffisant à elle-même et (parce que) vivant dans la terre / un homme diminué, "sans mains " ${ }^{30}$, impuissant, errant sous la pluie. N'est-ce pas cette force initiale d'une femme capable de faire quelque chose toute seule, dans le trou qu'elle a creusé, cette force qu'on lui a arrachée en brisant son intimité avec la terre, que traquent et tentent d'orienter tous les rites qui font du corps des femmes leur instrument?

On notera aussi que l'opposition initiale entre les sexes n'est pas gommée par la construction de la maison et l'hospitalité finale de l'homme. Car si la femme est contrainte d'accepter la cohabitation, il n'est pas dit qu'elle accepte
30 L'expression « sans mains " renvole au terme désignant un nomme " complet », kabanan, et, par extension, en référence au nombre de doigts des mains et des pieds, le chiffre "vingt ». 
pour autant que l'homme puisse désormais voir « ce qu'elle fait ». Les femmes désertent toujours l'espace domestique en ces moments particuliers où elles saignent, accouchent ou sacrifient.

L'activité sacrificielle des femmes peut être alors reconsidérée sous un autre jour, non plus comme une reprise ou une reproduction de l'accouchement et de la naissance, mais bien comme un acte de séparation d'avec une matrice originelle d'où vient un pouvoir procréateur dont, loin d'être les détentrices " naturelles", elles ne sont que les dépositaires. Pour que leur soit renvoyé, à chaque nouvelle gestation, ce pouvoir, il fallait que soit brisée cette intimité initiale avec la terre et ses puissances.

Des questions posées par Michel Cartry, qui font retour chaque fois qu'on relit ses textes ou que l'on tente d'ébaucher quelque réponse à celles par lui un jour énoncées, la fin est loin d'être signée. 


\section{Références bibliographiques}

Adler, A.

2010 "Logique sacrificielle et ordre politique: le statut de la personne du chef en relation avec son statut de sacrifiant ", dossier "Le Chemin du rite. Autour de l'œeuvre de Michel Cartry ", Revue Incidence, nº 6, pp. 149-160.

Cartry, M.

1978a "Les yeux captifs ", in Systèmes de signes. Textes réunis en hommage à Germaine Dieterlen, Paris, Hermann, pp. 79-110.

1978b "Le statut de l'animal dans le système sacrificiel des Gourmantché, deuxième partie ", Systèmes de Pensée en Afrique Noire, Le Sacrifice II, cahier 3, pp. 55.

1989-90 "Conférences", Annuaire de l'École pratique des hautes études, Section des sciences religieuses, tome 98 , année 1989.

1992 "From one rite to another: the memory in ritual ant the ethnologist's recollection ", in D. de Coppet (ed.), Understanding Rituals, Londres, Routledge.

2006 [Version française] : « D’un rite à l'autre: la mémoire du rituel et les remémorations de l'ethnologue ", Incidence 2, pp. 155-168.

2009 "De la divination au sacrifice: la métaphore de l'attache ", in M. Cartry, J.-L. Durand, R. Koch-Piettre (dir.), Architecturer l'invisible. Autels, ligatures, écritures, Brepols, BEHE, Sciences religieuses, n 138.

Cartry, M. \& Liberski, D.

1990 "Fondation sans fondateur ", Tracés de fondation, M. Détienne (dir.), Louvain-Paris, Peeters, Bibliothèque de l'École des Hautes Études, Sciences religieuses, vol. XCIII.

Diatta, N.

1998 Proverbes jóola de Casamance, Paris, Karthala.

Journet-Diallo, O.

1993 "Le harpon et le bâton (Joola Felup, Guinée-Bissau) ", Systèmes de pensée en Afrique Noire, 12, Fétiches : puissance des objets, pp. 17-37.

2009 "Prendre un bákiin sur le dos": destins et transmission des aires sacrificielles en pays jóola (Sénégal/Guinée-Bissau) ", in M. Cartry, J.-L. Durand, R. Koch-Piettre (dir.), Architecturer l'invisible. Autels, ligatures, écritures, Brepols, BEHE, Sciences religieuses, $n^{\circ} 138$.

Marcel, M. \& Hubert, H.

2000 [1899] "Essai sur la nature et la fonction du sacrifice ", CEuvres, tome 1: Les Fonctions sociales du sacré, Paris, éd. de Minuit, pp. 193-352.

Schloss, M. R.

1988 The Hatchet's Blood, Separation, Power, and Gender in Ehing Social Life, Tucson, University of Arizona Press, 178 p. 
Turner, V. W.

1967 The Forest of Symbols: Aspects of Ndembu ritual, Ithaca, Cornell University Press, 1967.

Zempleni, A.

1991 "Initiation ", in A. Bonte \& M. Izard, Dictionnaire de l'ethnologie et de l'anthropologie, Paris, PUF, pp. 375-377. 